\title{
Enhancing Apprentice-Based Learning of Java
}

\author{
Michael Kölling \\ Mærsk McKinney Moller Institute \\ University of Southern Denmark \\ mik@mip.sdu.dk
}

\author{
David J. Barnes \\ Computing Laboratory \\ University of Kent \\ D.J.Barnes@kent.ac.uk
}

\begin{abstract}
Various methods have been proposed in the past to improve student learning by introducing new styles of working with assignments. These include problem-based learning, use of case studies and apprenticeship. In most courses, however, these proposals have not resulted in a widespread significant change of teaching methods. Most institutions still use a traditional lecture/lab class approach with a strong separation of tasks between them. In part, this lack of change is a consequence of the lack of easily available and appropriate tools to support the introduction of new approaches into mainstream courses.

In this paper, we consider and extend these ideas and propose an approach to teaching introductory programming in Java that integrates assignments and lectures, using elements of all three approaches mentioned above. In addition, we show how the BlueJ interactive programming environment [7] (a Java development environment aimed at education) can be used to provide the type of support that has hitherto hindered the widespread take-up of these approaches. We arrive at a teaching method that is motivating, effective and relatively easy to put into practice. Our discussion includes a concrete example of such an assignment, followed by a description of guidelines for the design of this style of teaching unit.
\end{abstract}

\section{Categories and Subject Descriptors}

K.3.2 [Computers \& Education]: Computer \& Information

Science Education - Computer Science Education

D.1.5 [Programming Techniques]: Object-Oriented

Programming.

General Terms: Pedagogy, course design.

Keywords: Pedagogy, Objects-First, Java.

\section{INTRODUCTION}

Most introductory computing courses follow a roughly similar organizational structure: a sequence of weekly lectures is complemented by laboratory classes. The lectures are used to introduce new material to the students, and the lab classes reinforce the material by requiring the students to work through and discuss small exercises and somewhat larger assignments.

Permission to make digital or hard copies of all or part of this work for personal or classroom use is granted without fee provided that copies are not made or distributed for profit or commercial advantage and that copies bear this notice and the full citation on the first page. To copy otherwise, or republish, to post on servers or to redistribute to lists, requires prior specific permission and/or a fee.

SIGCSE'04, March 3-7, 2004, Norfolk, Virginia, USA.

Copyright 2004 ACM 1-58113-798-2/04/0003 ..\$5.00.
Intriguingly, despite numerous changes in programming languages, and shifts in programming paradigm over the last couple of decades, this delivery pattern has changed little and remains the predominant one in many institutions.

For teachers and students alike, assignments are key to the success of the learning process. In particular, learner-centred tasks [9] that capture the interest of students are more likely to generate a sense of excitement and motivate further investigation. Here, programming projects with a real purpose and interesting goals can be carried out. If done well, the results can be both enlightening and rewarding.

However, several problems exist in providing sufficiently motivational assignments:

- It is not easy to see how students can work on problems large enough to be truly interesting early in the course, while they have little experience with software development.

- It is often hard to create an obvious connection between the lecture and the assignment. Both often exist as fairly separate activities, making it harder to create interest in and motivation for the lectures.

- Programming environments are often either overly complex, incomplete in their language support, or do not provide good support for the teaching and learning processes, thus hindering active assignment work early in the course.

In this paper, we discuss a technique that can be used to integrate assignments and lectures more tightly. This serves to better motivate lecture content, results in the ability to carry out more interesting assignments and allows inclusion of important software engineering concepts into an introductory course.

Practical application of this technique for teaching introductory Java is facilitated by the availability of the BlueJ interactive programming environment. Unlike professional Java programming environments, BlueJ is specifically designed for the teaching and learning of key object-oriented concepts, but at the same time BlueJ supports the full implementation of the Java language and not just a subset.

\section{PREVIOUS WORK}

In the 1990 s, several related tracks were followed in an attempt to find more effective ways of motivating and presenting material on introductory programming courses.

Seminal among these attempts was the work of Linn and Clancy [8], who made a strong argument for the use of case studies to 
support program design. Particularly effective in their study was the use of expert commentary to accompany a design.

Also significant was the work of Astrachan and Reed [2,3] whose applied apprenticeship-approach encouraged students to read, study, modify and extend programs written by experienced programmers.

One of the principles of the apprentice-based approach is that it is particular applications that are the motivation for introducing new programming constructs or data structures, rather than studying constructs as an end in themselves. A similar problem-driven motivation can be found in the use of problem-based learning environments $[4,5]$ which also often feature group working.

Yet, despite the progress made in the early 1990s by work such as this, most modern introductory programming text books still tend to exhibit mainly traditional characteristics of language-construct driven chapters, and small example problems. Why is this?

Within the Java community, the easy availability of GUI-based examples was seen as one of the main means to motivate students [10]. Unfortunately, while these may well have motivational potential, there remains the question of whether - of itself - this is enough to deliver the broader educational and software engineering requirements of an introductory programming course.

As a consequence, there is a good case for revisiting and extending this earlier work in order to support a high quality introduction to object-oriented programming.

\section{PROBLEMS WITH ASSIGNMENTS}

In many courses assignments tend to be somewhat removed from the lectures in both organization and content. Usually, a lecture introduces new programming constructs or techniques and later, an assignment is given to practice application of these techniques. The larger (and with it, the more interesting) the assignment is, the more removed it tends to be from the lecture content, since it includes material covering a longer period of time.

Another problem with early programming assignments is that it is hard to get students to do things well. While students are struggling with getting their program to do something at all, they often have little time left for thinking about non-functional aspects, such as structural software quality.

The solution in many courses is to leave software quality aspects, such a maintainability, coupling and cohesion, to later courses, and concentrate on getting something running first.

This is unfortunate and we would like to incorporate critical assessment and evaluation of existing code into the curriculum very early on.

\section{OUR GOALS}

Our goals are twofold: firstly, we want to use a more problemdriven approach than the traditional style. The problem-driven approach presents a practical programming problem first, followed by the examination of possible solutions, possibly by introducing new programming constructs or techniques. This both ties the assignment and lecture close together, and provides a motivation for the introduction of new lecture material.
In fact, the role of lecture and assignment is reversed: it is not the lecture content that drives the assignment, but the assignment problems that drive the lectures.

Secondly, we want to achieve the inclusion of modern software engineering tasks into the computing curriculum early on. Traditionally, early computing assignments often use a blank screen approach: students start with nothing more than a problem specification. They then start designing and coding a new application from scratch. The essential assignment task is to write code.

This style does not reflect realities in the contemporary computing industry, where tasks like reading and understanding of existing code, maintenance and refactoring, adaptation and extension are far more common than the development of new applications.

We would like to emphasize that critical code reading and maintenance are essential skills for any programmer let loose on the world today.

Thus, this proposal affects both the form and the content of the material used in lectures and assignments. While our discussion of previous work shows that neither of these goals is new in itself, experience shows that implementation of them has been slow.

In part, this is because it has often remained difficult to put these ideas into practice. Linn and Clancy [8] noted, for instance, that students often found lengthy expert commentaries difficult to read, while the familiar syntactic hurdle of Java's main method almost forces an early focus on syntax that may be hard to break away from.

With the wide acceptance of Java, libraries and tools are now available that may help in supporting these approaches and make it worthwhile to revisit these issues. We hope to present a very practical, easily realizable example of how these goals can be achieved.

We aim to do this on two levels: firstly, by presenting one concrete example; and secondly, by presenting abstract guidelines for the development of such teaching units in general.

\section{A CONCRETE EXAMPLE}

\subsection{Exploration}

The example we discuss here to illustrate our approach is called The World of Zuul. When introduced to the students, the application compiles and executes.

The first student activity is to explore and describe the application. This exploration takes place within the BlueJ environment (Figure 1) and includes discussion of functional aspects (What does the program do?) and implementation aspects (What is the role of each class in the application?).

Interactive exploration is enhanced significantly by the features provided by BlueJ, such as the UML visualisation of the application structure, interactive object creation and method calling, object-state inspection and source-code exploration.

Students quickly find out that the application implements a framework for an adventure game [1] that allows the player to enter text commands and move around between a small number of locations (using commands such as go east). 
Exploration of the implementation is done as a group activity, in which students examine the classes' source code and explain each class in turn to other group members. The classes are well commented, so that most of the important information is easily accessible without the need to understand all details of the code.

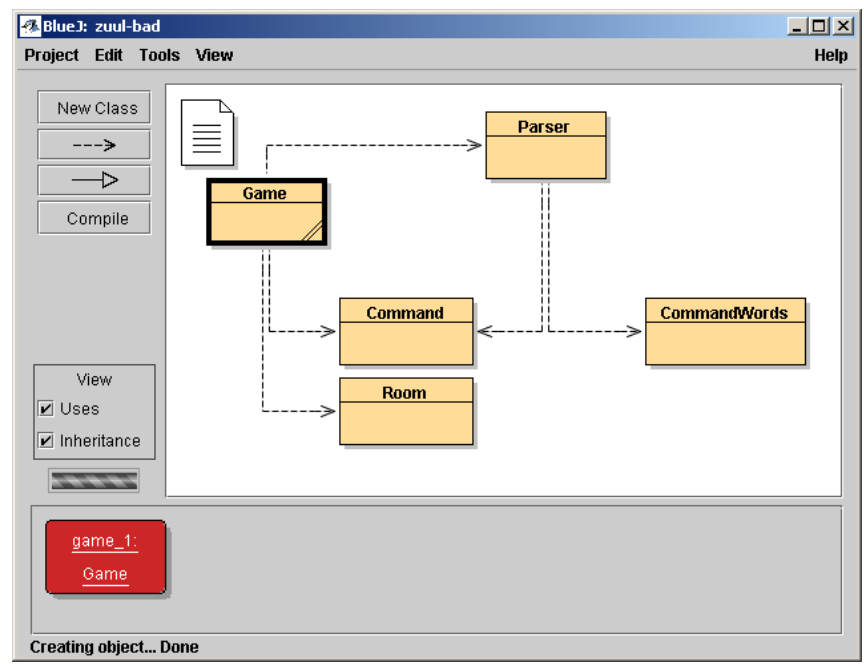

Figure 1: The World of Zuul project within BlueJ

\subsection{The first tasks}

It is clear to the students from their exploration that the game in its current form is rather limited in its functionality, and that it needs to be extended to turn it into a real game.

The next thing the students do is to invent an alternative game scenario. This can be done using a large variety of interactive or group activities, in which students develop and discuss ideas, finishing with every student selecting and describing a game plan. The explorations do not need to be constrained by implementation considerations. Topics can be anything: blood cells travelling through the human body; "you are lost in the shopping mall" themes; or the typical dungeon and dragon style scenarios.

Next, a number of small improvements to the given application are discussed. These are the addition of new movement directions (up and down), introduction of items in rooms (initially only one item per room) and appropriate new commands, such as 'take' and 'drop'.

\subsection{Discussion}

These first small tasks are discussed in detail in a lecture, including interactive development of an improved solution from the original limited version.

Even without the students having written a single line of code, it is clear that a number of important topics have been explored and practiced, such as code reading, and abstracting from the details of a particular game to its general characteristics.

Discussion of the necessary changes to the source code to attempt the first extension fits the model of learning from experts [2, 8] but with an important difference. Discussion quickly moves to code quality and it becomes obvious that the given code makes these simple extensions quite hard, because it is badly structured. This gives us the opportunity to discuss aspects of code that make maintenance easy or difficult.
We discover cases of code duplication, broken encapsulation and bad distribution of responsibilities, and we see how these make our life harder. Developing an ability to evaluate code critically is key here.

This gives us the further opportunity to discuss the fact that the functional view does not tell us about the quality of the underlying code (the program 'worked', after all).

Students often struggle with the idea that they received a low mark because their program appeared to do all that was required of them, but it was badly implemented. From here, we can illustrate refactoring, introduce concepts of coupling and cohesion, and goals such as localization of change.

We improve the design first by refactoring relevant bits of code, and then we find that making our intended extensions can become quite easy.

Thus, the first task (adding up and down movement) is solved completely in the lecture, with extensive discussion about considerations of code quality while making modifications to the given source.

\subsection{Exercises}

A second group of tasks (e.g., adding items to rooms) is done as a series of exercises. The problem and some aspects of a solution are discussed, and then students are expected to implement the detailed solution on their own.

The discussion contains a hint to the solution, and asks the relevant questions to make students consider important aspects; 'We have discussed responsibility-driven design - which class should be responsible for printing out the details of the items present in a room? Why?'

The exercises are organised in a sequence of manageable steps in increasing complexity. Adding items to rooms, for example, is initially done by supporting at most a single item being placed in each room, and then extended in a separate exercise to rooms holding an arbitrary number of items. Accordingly, the player can initially pick up and carry a single item, which is later extended to a set of items. The number of items carried can later be limited by a maximum possible weight that a player can carry.

\subsection{Assignment}

The exercises then lead into a larger assignment. In this assignment phase students implement their own game scenario including their own ideas for making the game interesting.

Typical elements students implement include forms of time limits, magic transporter rooms, trap doors, locks, talking characters, moving characters, and more.

At this stage, students receive less help and guidance in developing their solutions than during the exercise phase. They are expected to develop solutions on their own, with the possibility to ask a tutor for support.

During this phase, tutors frequently discuss the quality of students' solutions under maintainability and extendibility aspects with the student. 
It is known that code quality, reviewed under the aspects discussed in the lecture, will be a major component in the marking scheme for the assignment.

\section{THE THREE STEPS}

The previous section described a specific example of an assignment following our approach. In this section, we discuss the ideas behind this structure in a more general form.

On the first level, the assignment approach can be divided into three steps: Observation, Application and Design.

(Note that the order of activities is exactly reversed compared to classical, clean slate assignments: there, students typically have to start with design, followed by application, before they observe behaviour.)

\subsection{Step 1: Observation}

In this step, the instructor demonstrates a software engineering task actively in the lecture. This part is modelled on the apprentice approach: students observe the instructor performing a relevant task and listen to the instructor's commentary, while having the opportunity to interrupt and ask questions.

Aspects of this phase are typically the analysis of given code and the discovery of problems and ideas for solutions. It gives an opportunity to reflect on existing code and to evaluate critically before making changes.

Typically, the problems discovered during the evaluation of the code lead to a motivation for new course material, which can then be introduced and discussed. Students then observe the application of the new material in a well-chosen example, with the opportunity to discuss alternatives.

\subsection{Step 2: Application}

The educational goal of the second step is the application of new material under guidance.

Teachers discuss selected problems, chosen to display similar challenges to those demonstrated in step one, and give hints to solutions. Problems are chosen so that variations of the material from step one are applicable for the solution.

Students are expected to mirror the critical analysis and evaluation activities of their teacher and actively reason about the given code and argue about intended solutions.

This phase usually spans an arbitrary mix of lecture and lab classes, and alternates repeatedly between active coding activities and reflective discussion.

\subsection{Step 3: Design}

In the third step, students design their own tasks as extensions of the project at hand. It is a free programming assignment that allows students to apply all the techniques they are familiar with at that stage.

Typically, students are given a minimum of guidance on expected tasks, simply to communicate the required amount of work for marking purposes. At this stage, this only includes the description of sample tasks, not usually pointers to solutions.
One of the advantages of doing an assignment task in this context is that students are familiar with the framework they are expected to extend. Since steps one and two have served to familiarize the student with a given application, larger, more complex and more interesting applications can be used.

Extension tasks proposed by students are typically reviewed and guided by a tutor to ensure their usefulness in applying interesting course material, and their suitability in workload and level of difficulty.

\section{IMPORTANT ASPECTS OF THIS APPROACH}

\subsection{Problem driven}

The introduction of new material is driven by a concrete problem. The motivation for introduction of new concepts comes from a concrete task at hand.

This can easily be combined with additional problem-based learning approaches, such as student-controlled discovery of new material. Instead of presenting all new constructs in a lecture, students can be guided towards resources that enable them to discover new material as part of a student activity.

\subsection{Apprentice approach}

Our approach is an extension of the apprentice approach. Students start by studying expert written code: both well-written code and code to be critically evaluated and improved under expert guidance.

An important part of students' learning comes from experiencing an expert in action, hopefully imitating some of the activities considered good practice in their own work. This activity should be facilitated by a Java environment that supports incremental development and testing.

One of the important additions to the original apprentice approach as described in [2] is that students can also observe the process of the expert's work, in addition to the created artefact.

\subsection{Open / closed}

It is important to have characteristics of both open and closed assignments. The task should be well enough described so that weaker or less enthusiastic students have clear guidance as to what is expected of them, and how much they have to accomplish to receive a satisfactory mark in the assessment.

On the other hand, the task should be open enough that students can incorporate their own ideas and progress much further than the minimum required pass level.

It is common in computing classes that student groups display a wide variety of skills, and making the task challenging and interesting for even the best students is an important goal. This encourages both creativity and innovation.

\subsection{Ownership}

Whenever possible, the problem should be set in such a way that the student can take ownership of the task. In the Zuul example, this is achieved by letting students invent and design their own game scenarios and individual extension tasks. This, in turn, is the 
result of their abstracting from a particular example to the general principles it embodies, and on to a further instantiation.

From that moment on, when students work on the implementation of the task, they don't view it so much as work on a problem outside their control, but as implementing their game.

\subsection{Student controlled}

Another related (but distinct) issue is the ability of a student to take control over significant parts of the task.

Game-based assignments have been discussed in the past in the context of gender bias [6]. Studies have found that female students are often interested in different kinds of computer games than male students, and that games without any social component or relevance are less likely to engage female students.

While The World of Zuul is clearly a game-based example, we have not observed the described gender effect in its use. (While we have not carried out a formal investigation, we have consciously monitored this aspect and held informal talks with students about it.)

We speculate that the reason for this is that students can individually decide the context of their tasks by inventing their own scenarios. Giving students this degree of control might lead to higher acceptance of the relevance of the task.

\section{SUMMARY}

Despite the evident educational and motivational value of problem-based approaches to introductory computer science, the traditional delivery style involving separation of lecture material and lab material continues to dominate in many places.

In this paper we have described a concrete example of a more integrated approach that can be used in introductory Java courses. It includes problem-driven aspects, but is easier to realise than a complete problem-based learning model.

In addition, we have provided guidelines to assist others to apply this approach to their own material - perhaps in an even broader context than simply introductory Java teaching. This approach fosters a concept-driven approach to delivery of new material, and encourages ownership of these concepts by the students.

\section{REFERENCES}

[1] Adams, R., The Colossal Cave Adventure, web site, accessed 12 September 2003, http://www.rickadams.org/adventure/.

[2] Astrachan, O. and Reed, D., AAA and CS 1: The Applied Apprenticeship Approach to CS 1, Proceedings of SIGCSE 27 (March 1995).

[3] Astrachan, O., Smith, R., and Wilkes, J., Application-based Modules using Apprentice Learning for CS 2, Proceedings of SIGCSE 1997 (March 1997), 233-237.

[4] Barg, M., Fekete, A., Greening, T., Hollands, O., Kay, J., Kingston, J.H., Problem-based learning for foundation computer science courses, Computer Science Education 10 (2000), 1-20.

[5] Ellis, A., Carswell, L., Bernat, A., Deveaux, D., Frison, P., Meisalo, V., Meyer, J., Nulden, U., Rugelj, and J., Tarhio, J., Resources, Tools, and Techniques for Problem Based Learning in Computing, Proceedings of ITICSE '98 (August 1998), 46-50.

[6] Inkpen, K., Upitis, R., Klawe, M., Lawry, J., Anderson, A., Ndunda, M., Sedighian, K., Leroux, S., Hsu, D., "We Have Never Forgetful Flowers in Our Garden:" Girls' Responses to Electronic Games, Journal of Computers in Math and Science Teaching 13 (1994), 383-403.

[7] Kölling, M., Quig, B., Patterson, A. \& Rosenberg, J., The BlueJ system and its pedagogy. In Journal of Computer Science Education, Special issue on Learning and Teaching Object Technology, Vol 13, Nr 4, December 2003

[8] Linn, M.C., and Clancy, M.J., The Case for Case Studies of Programming Problems, Communications of the ACM (March 1992), 121-132.

[9] Norman, D.A., and Spohrer, J.C., Learner-Centered Education, Communications of the ACM 39 (April 1996), 24-27.

[10] Reges, S., Conservatively Radical Java in CS1, Proceedings of SIGCSE 2000 (March 2000), 85-89. 